\title{
A Complete System of Wireless Power Transfer Using a Circularly Polarized Retrodirective Array
}

\author{
Mohammad Fairouz ${ }^{1, *} \cdot$ Mohammad A. Saed $^{2}$
}

\begin{abstract}
A complete system of wireless power transfer using a circularly polarized retrodirective array is presented. A dual frequency, active retrodirective array is proposed for a transmitter system. The antenna array uses circularly polarized microstrip patch antenna subarrays with sequential rotation and surface wave suppression. The designed antenna element eliminates undesired coupling between array elements due to surface waves present in conventional microstrip antenna arrays in order to improve array performance. A sequential rotation technique was implemented to improve impedance matching and circular polarization bandwidths. The proposed retrodirective array was designed to operate at about $2.4 \mathrm{GHz}$ for the interrogating signal and about $5.8 \mathrm{GHz}$ for the retransmitted signal. The beam scanning inherent in retrodirective arrays ensures a uniform power level available to the receiving devices, regardless of their location within the angular sector over which retrodirectivity is achieved. A rectenna was designed as a receiver in order to have a complete system the wireless power transfer. A zero bias Schottky diode with high detection sensitivity was used as the rectifying device. The shorting pins used in the antennas to suppress surface waves also act as return paths for the DC current, eliminating the need for an RF chock in the rectifier circuit. The design procedure, simulation results, and experimental measurements are presented.
\end{abstract}

Key Words: Microstrip Patch, Rectenna, Retrodirective Array, Sequentially Rotation, Wireless Power Transfer.

\section{INTRODUCTION}

In recent years, there has been considerable interest in wireless power transfer (WPT) and energy harvesting to charge devices without wires [1-6]. WPT methods can be broadly divided into two groups: a group operating over short distances relying primarily on reactive coupling [4-6] and a group operating over long distances relying on radiation mostly using $\mathrm{RF} / \mathrm{mi}^{-}$ crowave energy $[2,3]$. The most common methods employed in reactive coupling are based on magnetic field induction between two coils placed in close proximity to each other. In addition to charging wireless devices, WPT relying on inductive coupling to charge electric vehicles (EV) while moving was demonstrated in [5]. A technique using microwaves to transfer information and power was reported in [2]. Very low power transfer efficiencies encountered in radiation methods over long distances are of primary concern; improvements in these efficiencies have significant impact on practical implementations of such methods. Promising techniques to improve the power transfer efficiency are based on retrodirective arrays [7-10]. A retrodirective array is a smart antenna array that scans its main beam to point to the direction of a transmitter without prior knowledge of the transmitter's location. Successful implementation of retrodirective arrays in WPT systems ensures that all devices within a certain

Manuscript received September 27, 2019 ; Revised January 12, 2020 ; Accepted March 25, 2020. (ID No. 20190927-081J)

${ }^{1}$ The Higher Institute of Telecommunication and Navigation, Safat, Kuwait.

${ }^{2}$ Department of Electrical and Computer Engineering, Texas Tech University, Lubbock, TX, USA.

"Corresponding Author: Mohammad Fairouz (e-mail: ma.fairouz@paaet.edu.kw)

This is an Open-Access article distributed under the terms of the Creative Commons Attribution Non-Commercial License (http://creativecommons.org/licenses/by-nc/4.0) which permits unrestricted non-commercial use, distribution, and reproduction in any medium, provided the original work is properly cited.

(c) Copyright The Korean Institute of Electromagnetic Engineering and Science. All Rights Reserved. 
sector will receive uniform power density, unlike antennas with fixed beams. Due to this significant benefit, in this paper we focus on designing a WPT system based on an active retrodirective array as a transmitter. To further increase efficiency and reduce mutual coupling between array elements, the antenna array in our system uses microstrip elements with shorting pins $[11,12]$. Conventional microstrip antennas printed on dielectric substrates suffer from excitation of undesired surface waves. In addition to lowering the antenna efficiency and distorting radiation patterns, surface waves increase mutual coupling between the elements of the antenna array, which can lead to scan blindness. This can be a serious problem in retrodirective arrays since the antenna beam is scanned to point toward the interrogating signal. For demonstration purposes, a circularly polarized four-element antenna subarray using microstrip patches with shorting pins was designed and implemented in the transmitter of retrodirective array WPT system. To increase the axial ratio and impedance bandwidth of circularly polarized arrays, sequential rotation techniques can be used [13-16]. In our design, sequential rotation was used where the four elements in the subarray were rotated by $90^{\circ}$ in orientation and their input ports were excited with uniform magnitude and $0^{\circ}$, $90^{\circ}, 180^{\circ}$, and $270^{\circ}$ in phase difference. In addition to the retrodirective array transmitter, a rectenna using microstrip patches with shorting pins and zero-bias Schottky diode was designed and implemented as the receiver for charging devices in the WPT system. The shorting pins serve a dual role in the rectenna, they help suppress surface waves as in the transmitter array and they provide a DC patch for the diode's DC bias avoiding the need for an $\mathrm{RF}$ chock present in traditional rectenna designs.

\section{SYSTEM DESCRIPTION}

The retrodirective WPT system is illustrated in Fig. 1. The antenna array in the retrodirective $5.8 \mathrm{GHz}$ transmitter is comprised of two $2 \times 2$ sequentially rotated microstrip subarrays incorporating surface wave suppression using shorting pins. The subarrays are aperture-coupled to a feed network on another substrate in a two-layer configuration. The interrogating signal is received using a $2.4 \mathrm{GHz}$ two-element microstrip array. The $5.8 \mathrm{GHz}$ receiving rectenna that would be placed in the device being charged consists of a two-element microstrip array of microstrip patches with shorting pins. The interrogating signal $(2.4 \mathrm{GHz})$ received at the retrodirective array is mixed with a local oscillator (LO) signal in an up-converting mixer to produce a $5.8-\mathrm{GHz}$ transmitted signal. The design proposed in this paper differs from conventional phase conjugating retrodirective arrays, even though both use mixers. In conventional designs, the mixers are used as down-converters requiring a high $\mathrm{LO}$ frequency (equal to the sum of the RF and IF frequencies), and

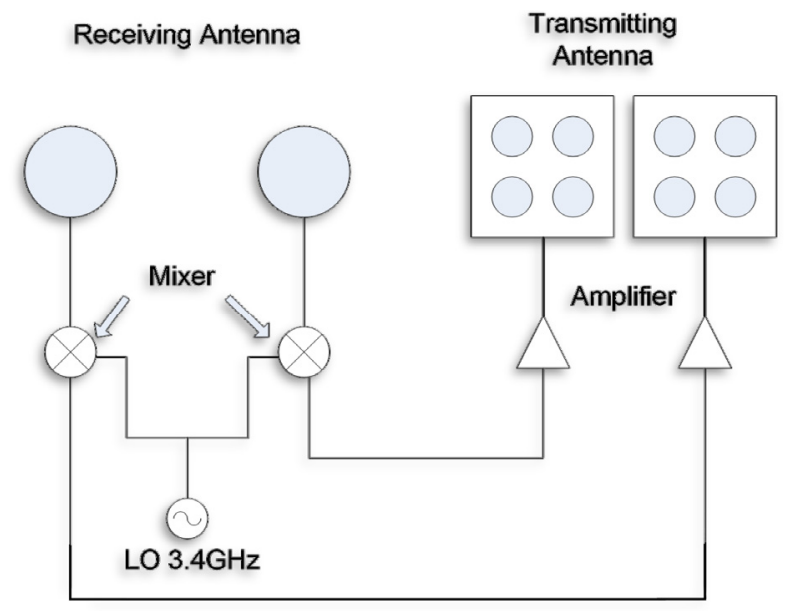

Fig. 1. Block diagram for the retrodirective array system.

therefore, the down-conversion results in phase conjugation of the signal needed for retrodirectivity. In our design, the mixers are used as up-converters, and therefore do not produce phase conjugation. The advantage is that a lower LO frequency is needed, equal to the difference between the RF and IF frequencies.

Retrodirectivity in our design is achieved by properly connecting the receiving and transmitting arrays to scan the transmitter beam in the direction of the interrogating signal, as in a Van-Atta array. In a basic Van-Atta linear array, receiving and transmitting antenna pairs are connected in such a way to retransmit the received signal towards the incoming signal. This is achieved by connecting the first receiving element to the last transmitting element, the second receiving element to the one before the last transmitting element, and so on. The transmission lines connecting receive/transmit pairs must be of equal lengths or plus or minus an integer multiple of a wavelength to ensure proper retrodirectivity. A plane wave incident on such an array with an angle of incidence $\theta_{i}$ induces voltages in the receiving elements with a progressive phase shift of $k d \sin \theta_{i}$, where $k$ is the propagation constant and $d$ is the element spacing. Due to the way receive/transmit pairs are arranged and connected, the signal will be retransmitted in the direction of $\theta_{i}$. In our design, since transmit and receive frequencies are different, the transmission line lengths in the feed network and transmit and receive array element spacing must be chosen properly to avoid beam pointing errors.

The transmission lines between the receiving array elements and the mixers must be equal, and the transmission lines connecting the mixers to the transmitting array elements must also be equal. This ensures that the phase shifts due to the connecting lines are equal for each transmit-receive antenna pair.

The circularly polarized microstrip patch antenna used in the transmitting subarrays was designed to suppress surface waves by incorporating four shorting pins. The positions of the short- 
ing pins are determined by numerically solving the following equation, presented in $[12,16]$ and repeated here for convenience:

$$
\begin{aligned}
& Y_{0}(k b)+Y_{0}\left(k d_{1}\right) \pm Y_{0}\left(k d_{2}\right) \pm Y_{0}\left(k d_{3}\right) \\
& -8 \sum_{n=1}^{\infty} x_{n} \cos ^{2}\left(\alpha J_{n}\left(\frac{k\left(r_{0}-b\right) J_{n}\left(k r_{0}\right) Y_{n}^{\prime}(k a)}{J_{n}^{\prime}(k a)}\right)\right) \\
& =0
\end{aligned}
$$

where $r_{0}$ is the pins' radial position, $b$ is the radius of the pins, $J_{n}$ and $Y_{n}$ are the Bessel functions of the first and the second kinds, respectively, $\alpha$ is the angle between the $\mathrm{x}$-axis and the pins (the feeding probe is located on the $\mathrm{x}$-axis), $x_{n}=$ $1(n \geq 1)$ for even mode and $x_{n}=1 / 2$ for odd mode, $d_{1}$, $d_{2}$ and $d_{3}$ are the distances to the pin at $\phi=+\alpha$ from the other three pins, given by $d_{1}=2 r_{0} \sin \alpha, d_{2}=2 r_{0}$, and $d_{3}=$ $2 r_{0} \cos \alpha$. The angle $\alpha$ is chosen equal to $45^{\circ}$ to maintain symmetry. Given a desired shorting pin radius $b$ and position $r_{0}$, Eq. (1) can be solved numerically for the required value of $k a$ to suppress the surface waves for the first order mode $\mathrm{TM}_{1}$. A graph for $k a$ versus pin position $r_{0} / a$ can then plotted, the curve is used to determine the pins' location. Circular polarization for each patch was obtained using a diagonal slot. Aperture coupling using rectangular slots in the ground plane was used to feed the antenna subarray with a feed network placed on another substrate, as shown in Fig. 2. Sequential rotation for the $2 \times$ 2 subarrays was also implemented to improve the axial ratio bandwidth, the fabricated subarray is shown in Fig. 3. The feeding network uses $\mathrm{T}$-junction power dividers with the appropriate characteristic impedances and line lengths to obtain the necessary phase shifts. The substrate used for the feed network is RT/Duroid 6010 with a dielectric constant $\varepsilon_{r}=10.2$ and thickness of $1.27 \mathrm{~mm}$, and the substrate used for the antenna is RT/Duroid 5870 with a dielectric constant $\varepsilon_{r}=2.33$ and thickness of $1.575 \mathrm{~mm}$. The complete array was simulated and

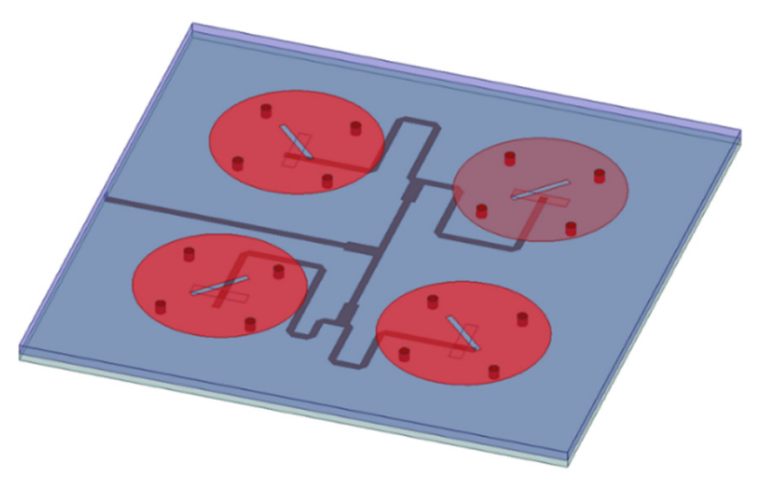

Fig. 2. A $2 \times 2$ sequentially rotated transmitting subarray (dimensions: top slot length $=10 \mathrm{~mm}$, top slot width $=1 \mathrm{~mm}$, patch radius $a=13.6 \mathrm{~mm}$, pin location $r_{0}=10.5 \mathrm{~mm}$, pin radius $b=0.8 \mathrm{~mm}$, bottom slot length $=9 \mathrm{~mm}$, and its width $W=1.5 \mathrm{~mm}$, element spacing $=40 \mathrm{~mm}$ ).
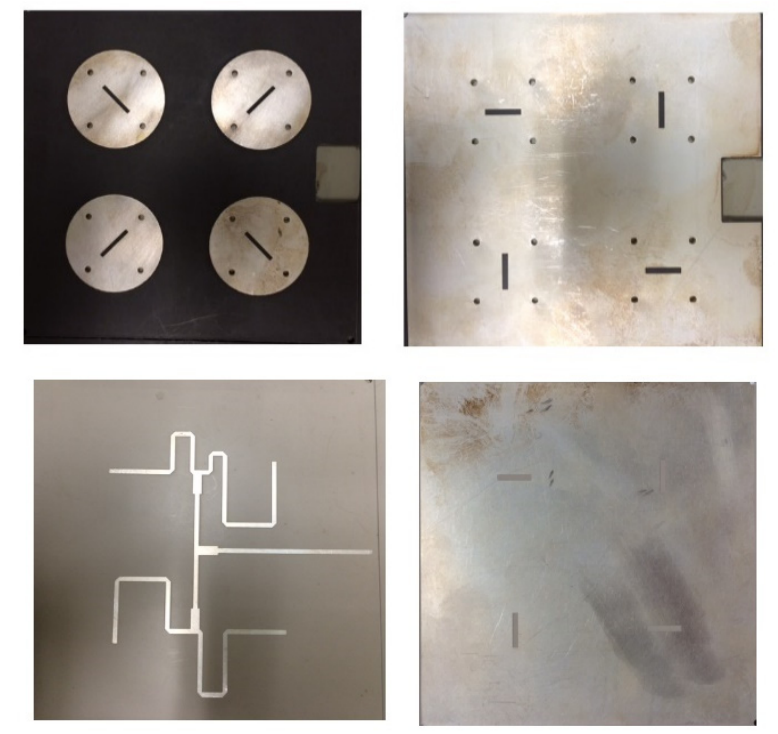

Fig. 3. The fabricated $2 \times 2$ transmitting subarray (top: front and back of the antenna substrate, bottom: front and back of the feed network substrate).

optimized in ANSYS HFSS, simulation and measurement results for the antenna array were presented in [16].

A rectenna was designed to demonstrate the wireless power transfer capability of the proposed system. A two-element antenna array, shown in Fig. 4, was used in the rectenna design. Each element is a probe-fed circularly polarized microstrip patch with shorting pins to reduce surface waves. However, sequential rotation was not implemented here since only two radiating elements were used for demonstration purposes. The two patches are combined using a $50-\Omega$ Wilkinson power divider, as shown in Fig. 5. It is worth noting that the shorting pins in the rectenna design serve to suppress surface waves and to provide a $\mathrm{DC}$ path in the rectifier circuit, avoiding the need for an RF choke used in typical rectenna circuits.

A microstrip bandstop filter was designed to pass $5.8 \mathrm{GHz}$ from the antenna to the diode and block the second harmonic at 11.6 GHz to improve efficiency [17]. The diode used in the rectenna design is a zero bias Schottky detector diode (HSMS-

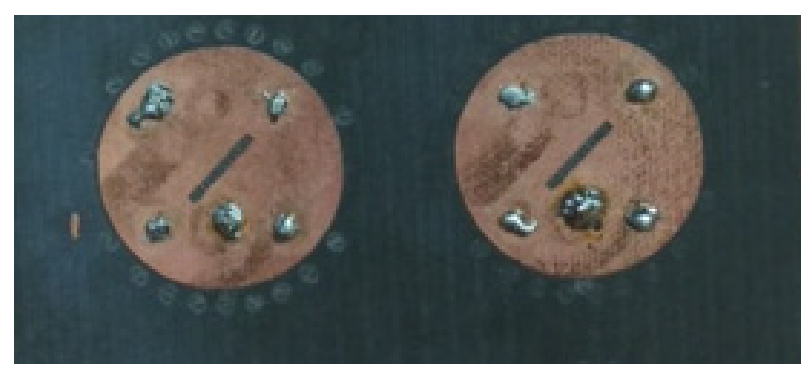

Fig. 4. The rectenna's two element array (dimensions: slot length $=$ $10 \mathrm{~mm}$, slot width $=1 \mathrm{~mm}$, patch radius $a=15.2 \mathrm{~mm}$, pin location $r_{0}=10.9 \mathrm{~mm}$, pin radius $=0.8 \mathrm{~mm}$, element spacing $=40 \mathrm{~mm}$ ). 


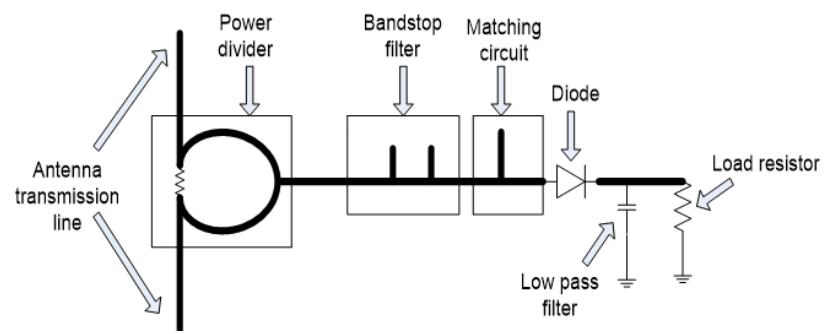

Fig. 5. The rectifier circuit.

2852) with sensitivity up to $25 \mathrm{mV} / \mu \mathrm{W}$ at $5.8 \mathrm{GHz}$. The rectifier circuit was optimized using Advanced Design System (ADS) software. The diode model parameters used in the simulation are as follows: series resistance $R_{s}=25 \Omega$, zero-bias junction capacitance $C_{j 0}=0.18 \mathrm{pF}$, breakdown voltage $V_{B}=3.8 \mathrm{~V}$, and maximum forward voltage $V_{F}=150 \mathrm{mV}$. The diode input impedance at $5.8 \mathrm{GHz}$ obtained through simulations is $Z_{d}=$ $13-j 33 \Omega$, a matching circuit using single open-circuited shunt stub was used to match $Z_{d}$ to $50 \Omega$.

\section{RESULTS}

A monostatic setup was used to test the performance of the retrodirective array, as shown in Fig. 6. Two fixed co-located antennas were used, one transmits the $2.4 \mathrm{GHz}$ interrogating signal and the other receives the retransmitted $5.8 \mathrm{GHz}$ signal. The retrodirective array was mounted on a rotational stage. A signal generator is connected to the $2.4 \mathrm{GHz}$ antenna to transm-
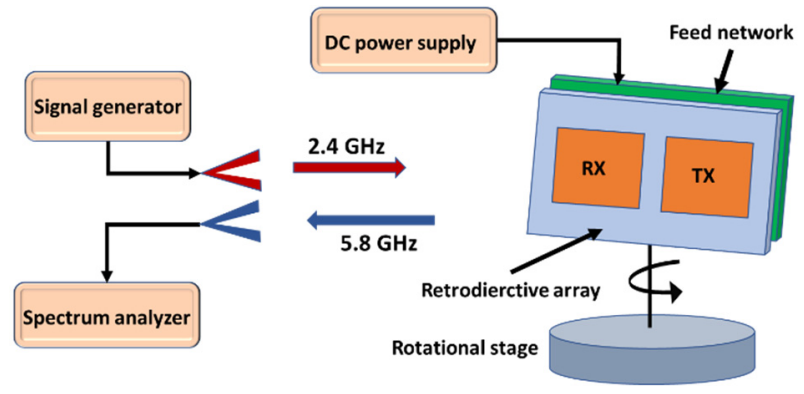

Fig. 6. Monostatic measurement setup.

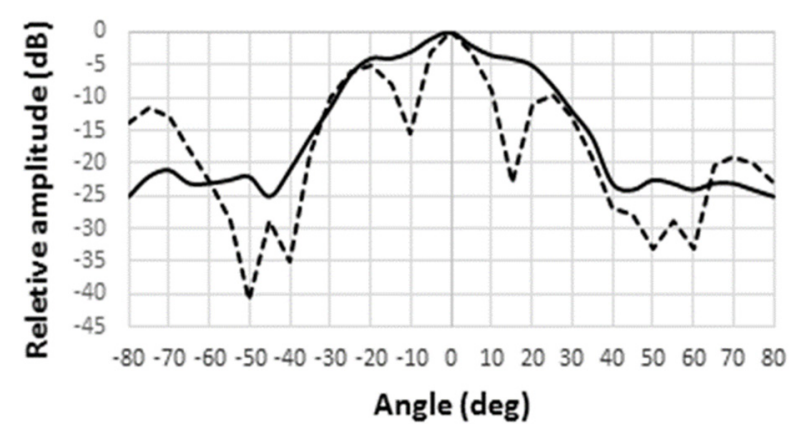

Fig. 7. Measured monostatic radiation patterns for retrodirective array (solid curve) and normal non-retrodirective array (dotted curve) at $5.8 \mathrm{GHz}$. it the interrogating signal, and a spectrum analyzer is connected to the $5.8 \mathrm{GHz}$ antenna to measure the power received from the retrodirective array. The measurement is performed at different angles by moving the rotation stage to record the power received at each angel. For comparison, the measurement is repeated using a normal non-retrodirective array in place of the retrodirective array. The normal non-retrodirective antenna array is comprised of the same sequentially rotated transmitting antenna portion of the retrodirective system. The radiation patterns (recorded by receiving antenna) was measured to test the system's capability in steering its beam toward the transmitter. The measured radiation patterns for both cases are shown in Fig. 7, the solid curve for the retrodirective array and the dotted curve for the normal array. Fig. 7 clearly shows the substantial improvement in the uniformity of the received power for the retrodirective array between $-25^{\circ}$ to $25^{\circ}\left(50^{\circ}\right.$ sector) versus the non-retrodirective array. In the normal non-retrodirective array, the radiation pattern shows that the received power starts to drop beyond $\pm 3^{\circ}$, and it has very deep nulls around $-10^{\circ}$ and $+15^{\circ}$ where the power is more than $12 \mathrm{~dB}$ and $17 \mathrm{~dB}$, respectively, below that of the retrodirective array.

It is also worth noting that the microstrip array element using shorting pins is larger than the conventional patch. This makes it difficult to keep the distance between the array elements half free-space wavelength. A separation more than $0.5 \lambda$ (but less than $\lambda$ ) results in higher side lobe levels in the radiation pattern and a narrower the beam.

The simulated reflection coefficient in decibels of the rectifier circuit, as seen at each antenna terminal (one of the output ports of the Wilkinson's power divider), is shown in Fig. 8. As shown in Fig. 8, excellent matching was achieved at $5.8 \mathrm{GHz}$, with a return loss better than $20 \mathrm{~dB}$. The RF-DC conversion efficiency versus input power obtained through simulations is shown in Fig. 9.

As shown in Fig. 9, the highest efficiency obtained is about $70 \%$ for an input power of $9 \mathrm{dBm}$. This efficiency compares favorably with those reported in [17-19] where efficiencies of

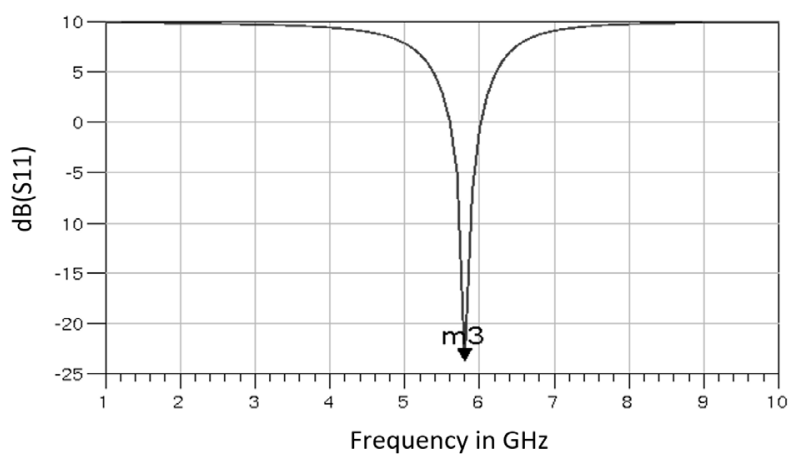

Fig. 8. Simulated reflection coefficient (in $\mathrm{dB}$ ) of the rectifier circuit. 


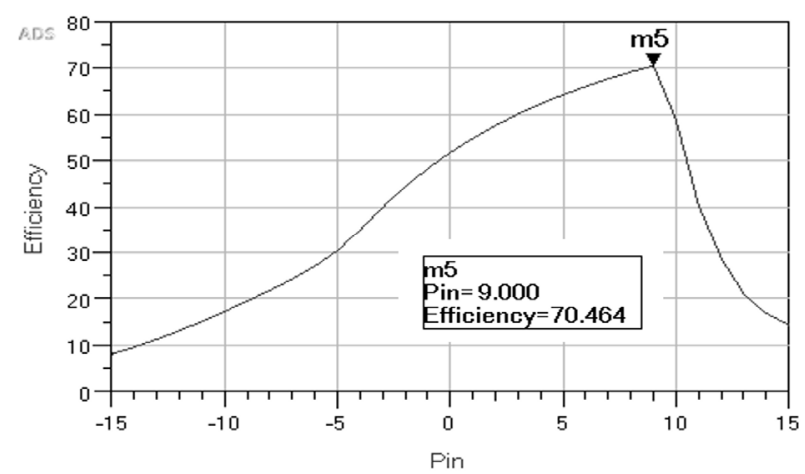

Fig. 9. Rectifier's conversion efficiency versus input power (in $\mathrm{dBm}$ ).

$68.5 \%, 60 \%$, and $65 \%$ were reported, respectively. A rectenna was also built and tested using a transmitting antenna placed 60 $\mathrm{cm}$ away from the rectenna. A DC output voltage about 200 $\mathrm{mV}$ was measured for received power of about $-10 \mathrm{dBm}$.

\section{CONCLUSION}

A complete wireless power transfer system using a circularly polarized retrodirective array was presented. The $5.8 \mathrm{GHz}$ transmitting array consisted of two sequentially rotated $2 \times 2$ subarrays comprised of circular microstrip patches with shorting pins to suppress surface wave and reduce mutual coupling between array elements. Up-converting mixers and amplifiers were used in the design. The retrodirective array was tested using a monostatic setup with a $2.4 \mathrm{GHz}$ interrogating signal. The measured results demonstrated that the implemented design substantially improves the uniformity of the power density available to the charging devices, regardless of their location within a $50^{\circ}$ angular sector. This beam scanning capability ensures a far more efficient power delivery than antennas with fixed beams to devices without prior knowledge of their location. A rectenna using a two-element microstrip antenna array was designed as a receiving device for the WPT system. The rectifier circuit was optimized to achieve high RF-DC conversion efficiency at 5.8 $\mathrm{GHz}$. The highest simulated conversion efficiency obtained for the proposed design was $70 \%$ for an input power of $9 \mathrm{dBm}$.

\section{REFERENCES}

[1] W. C. Brown, "The history of power transmission by radio waves," IEEE Transactions on Microwave Theory and Techniques, vol. 32, no. 9, pp. 1230-1242, 1984.

[2] T. D. P. Perera, D. N. K. Jayakody, S. K. Sharma, S. Chatzinotas, and J. Li, "Simultaneous wireless information and power transfer (SWIPT): recent advances and future challenges," IEEE Communications Surveys \& Tutorials, vol. 20, no. 1, pp. 264-302, 2018.

[3] S. T. Khang, D. J. Lee, I. J. Hwang, T. D. Yeo, and J. W. Yu,
"Microwave power transfer with optimal number of rectenna arrays for midrange applications," IEEE Antennas and Wireless Propagation Letters, vol. 17, no. 1, pp. 155-159, 2018.

[4] K. O'Brien, R. Teichmann, and H. Gueldner, "Magnetic field generation in an inductively coupled radio-frequency power transmission system," in Proceedings of 2006 37th IEEE Power Electronics Specialists Conference, Jeju, South Korea, 2006, pp. 1-7.

[5] D. Pital, K. Mcdonough, J. M. Miller, B. Fahimi, and P. T. Balsra, "Wireless power transfer for vehicular applications: overview and challenges," IEEE Transactions on Transportation Electrification, vol. 4, no. 1, pp. 3-37, 2018.

[6] R. Tseng, "Method and apparatus for wireless power transmission," U.S. Patent 9129741, Sep 14, 2007.

[7] V. Fusco, C. B. Soo, and N. Buchanan, "Analysis and characterization of PLL-based retrodirective array," IEEE Transactions on Microwave Theory and Techniques, vol. 53, no. 2, pp. 730-738, 2005.

[8] Y. Li and V. Jandhyala, "Design of retrodirective antenna arrays for short-range wireless power transmission," IEEE Transactions on Antennas and Propagation, vol. 60, no. 1, pp. 206-211, 2012.

[9] M. Fairouz and M. A. Saed, "A retrodirective array with reduced surface waves for wireless power transfer applications," Progress in Electromagnetics Research, vol. 55, pp. 179186, 2014.

[10] P. Chan and V. Fusco, "Co-operating retrodirective system" IET Microwaves, Antennas \& Propagation, vol. 7, no. 3, pp. 187-194, 2013.

[11] A. R. Al-Ajmi and S. F. Mahmoud, "A single-feed circularly-polarized patch antenna for reduced surface wave applications," Microwave and Optical Technology Letters, vol. 51, no. 11,pp. 2675-2679, 2009.

[12] S. F. Mahmoud and A. R. Al-Ajmi, "A novel microstrip patch antenna with reduced surface wave excitation," Progress in Electromagnetics Research, vol. 86, pp. 71-86, 2008.

[13] P. S. Hall, J. S. Dahele, and J. R. James, "Design principles of sequentially fed wide bandwidth circularly polarized microstrip antennas," IEE Proceedings H (Microwaves, Antennas and Propagation), vol. 136, no. 5, pp. 381-389, 1989.

[14] A. A. Kishk, "Performance of planar four elements array of single-fed circularly polarized dielectric resonator antenna," Microwave and Optical Technology Letters, vol. 38, no. 5, pp. 381-384, 2003.

[15] A. R. Weily and Y. J. Guo, "Circularly polarized ellipseloaded circular slot array for millimeter-wave WPAN applications," IEEE Transactions on Antennas and Propagation, vol. 57, no. 10, pp. 2862-2870, 2009.

[16] M. Fairouz and M. Saed, "A sequentially rotated circularly polarized microstrip antenna array with reduced mutual 
coupling," Electromagnetics, vol. 36, no. 7, pp. 422-433, 2016.

[17] J. Zbitou, M. Latrach, and S. Toutain, "Hybrid rectenna and monolithic integrated zero-bias microwave rectifier," IEEE Transactions on Microwave Theory and Techniques, vol. 54, no. 1, pp. 147-152, 2006.

[18] C. Chin, Q. Xue, and C. Chan, "Design of a 5.8-GHz rectenna incorporating a new patch antenna," IEEE $\mathrm{An}$ -

\section{Mohammad Fairouz}

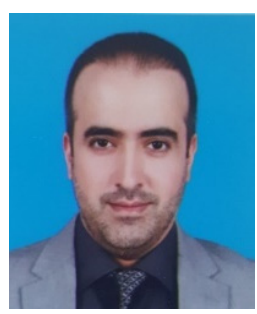

received his B.S. degree in electronic and communication engineering from Nottingham Trent University, Nottingham, United Kingdom in 2002, M.S. and $\mathrm{Ph} . \mathrm{D}$. degrees in electrical engineering from Texas Tech University in 2012 and 2015, respectively. He was a Value-Added Service (VAS) engineer at Ooredoo telecommunication company in Kuwait from 2002 to 2006. He joined the Higher Institute of Telecommunication and Navigation in Kuwait as an instructor in 2006 until now. His research interests are in Wireless power transfer, antenna, rectenna, microwave components and metamaterial. tennas and Wireless Propagation Letters, vol. 4, pp. 175-178, 2005.

[19] S. Ladan, N. Ghassemi, A. Ghiotto, and K. Wu, "Highly efficient compact rectenna for wireless energy harvesting application," IEEE Microwave Magazine, vol. 14, no. 1, pp. 117-122, 2013.

Mohammad A. Saed

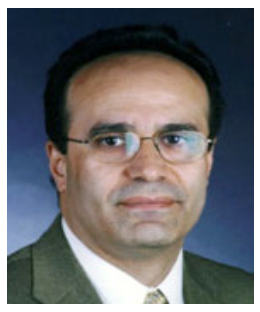

received his B.S. degree in electrical engineering from Middle East Technical University, Ankara, Turkey in 1983, and M.S. and Ph.D. degrees in electrical engineering from Virginia Tech, Blacksburg, Virginia in 1984 and 1987, respectively. From 1989 to 1990 , he was a research associate at Virginia Tech. In 1990, he joined the faculty of the Electrical Engineering Department of State University of New York, New Paltz. In 2001, he joined the Electrical and Computer Engineering Department at Texas Tech University in Lubbock, Texas where he is currently an Associate Professor. His research interests include applied electromagnetics with an emphasis on $\mathrm{RF} /$ microwave applications in imaging, sensing, manufacturing, computational electromagnetics, antennas, and microwave components. 